\title{
Empowering Low-Cost CMOS Cameras by Image Processing to Reach Comparable Results with Costly CCDs
}

\author{
Gözen Köklü • Julien Ghaye • Ralph Etienne-Cummings · Yusuf Leblebici • \\ Giovanni De Micheli • Sandro Carrara
}

Received: 14.08.2013 / Accepted: date

\begin{abstract}
Despite thehuge research effort toimprove the performanceof the Complementary MetalOxideSemiconductor (CMOS) image sensors, Charge-Coupled Devices (CCDs) still dominate the cell biology related conventional fluorescence microscopic imaging market where low or ultra-low noise imaging is required. A detailed comparison of the sensor specifications and performance is usually not provided by the manufacturers which leads the end users not to go out of the habitude and choose a CCD camera instead of a CMOS one. However, depending on the application, CMOS cameras, when empowered by image processing algorithms can become cost-efficient solutions for conventional fluorescence microscopy. In this paper, we introduce an application-based comparative study between the default CCD camera of an inverted microscope (Nikon Ti-S Eclipse) and a custom-designed CMOS camera and apply efficient image processing algorithms to improve the performance of CMOS cameras. Quantum micro-bead samples that emit fluorescence light at different intensity levels, breast cancer diagnostic tissue cell and Caco-2 cell samples are imaged by both CMOS and CCD cameras and results are provided to show the reliability of CMOS camera processed images and finally to be of assistance when scientists select their cameras for desired applications.
\end{abstract}

Keywords fluorescence microscopy cameras · CMOS camera - CCD camera · CCD vs CMOS - CMOS image sensor $\cdot$ CCD image sensor

\section{G. Köklü}

Integrated Systems Laboratory (LSI), Swiss Federal Institute of Technology (EPFL)

Tel.: +41-21-6938168 E-mail: gozen.koklu@epfl.ch

\section{INTRODUCTION}

Historically, CCDs have dominated the imaging sensors market. Today, the market share for CMOS image sensors is increasing and even surpassing CCDs in terms of volume [1]. However, CCDs are still the dominating technology for high quality imaging market and are used for high cost imaging applications such as microscopy for life and materials science applications in both clinical and educational domains. In the literature, there are many examples of use of CCD cameras for detecting fluorescent labelled Deoxyribonucleic Acid DNAs or some expressions on the stained, fixed or live cells. Some examples to that is imaging of growing DNA chains [2], real-time detection of DNA hybridization to DNA microarrays [3], monitoring of anticancer effects of some specific agents [4], examining of cell polarity on stained, fixed and live cells [5] and obtaining quantitative information about the chromatinDNA distribution inside the nucleus [6], [7], [8].

On the other hand, CMOS image sensors were mostly used in low performance devices (e.g. toys, cell phones [9], [10]) due to their inherent advantages such as low power consumption, low cost, compactness and high integration. Recently, this traditional misconception started to dissolve and CMOS imagers started to show up in both high quality Digital Single Lens Reflex (DSLR) cameras and biological applications. A couple of examples of CMOS cameras in biological applications include miniaturized fluorescence cameras for brain imaging [11], [12], [13] and fluorescence lifetime imaging with CMOS Single Photon Avalanche Diodes (SPADs) [14], [15] where CMOS sensor speed advantage becomes crucial. However, the use of CMOS based cameras in microscopy is still very limited although a CMOS imager can perform as well as a CCD imager on various grounds, depending on the application specifications while usually costing less than 
CCDs. To do that, it is of great importance to mitigate the downside of CMOS sensors, especially higher noise, with image processing algorithms dedicated for quantitative fluorescence microscopy measurements [16], [17], [18] and benefit from synthetic image generation techniques [19], to facilitate the progress on this domain.

Inthispaper, wetargetfluorescent detectionsystemsin upright or inverted microscopes due to their popularity in cell-level biology and biochemistry for a variety of experimental, analytical, and quality control applications asdescribed earlier. With thecomparativestudydescribed in the following sections, we show that even a midperformance CMOS camera when empowered by image processing algorithms can reach similar results with a widely used high-cost CCD camera results. This paper intends to show the potential use of CMOS cameras for microscopic applications, suggests thescientists to further diagnose their camera options before buying cameras and proposes different image processing methods that can be applied to reduce different types of noise as well as to make an easy comparative study.

The structure of this paper is as follows: in Section II, we describe the materials used in this experiment: the custom-designed CMOS camera, commercially available Nikon CCD camera and image intensity calibration kits, in Section III, we present the methods that are used in this experiment: image processing algorithms including noise reduction algorithms, auto-thresholding and image registration and resizing and the use of image intensity calibration kits to calculate the relative intensities of each kit sample from the collected camera images, in Section IV, the results are presented based on the calculation of the relative intensities as well as the detection of the morphological patterns on the tissue samples for cancer diagnostics and Caco-2 cell lines by both cameras.

\section{MATERIALS}

\subsection{Custom-Designed CMOS Camera vs Default CCD Camera}

In order to show the possible use of CMOS cameras for celllevel biological applications, we perform an applicationbased comparative study based on fluorescence imaging between the images collected from the default CCD camera of the Nikon Eclipse Ti (Nikon Instruments, Inc. Melville, N.Y.) inverted microscope and the customdesigned CMOS camera. The chosen CCD camera has been widely sold by Nikon for conventional microscopy applications and the custom-designed CMOS camera is assembled by using a mid-performance CMOS imager.
Since the focus of this paper is to introduce a lowcost replacement of standard CCD cameras, Scientific CMOS (sCMOS) cameras, which are the high-cost, newgeneration CMOS cameras providing a comparable and evenbetter performancethan Electron Multiplying Charge Coupled Devices (EMCCDs), are excluded from this study and instead the standard CMOS camera images are empowered by image processing algorithms.

Fig. 1 represents a block diagram of the entire fluorescence imaging system with the custom-designed CMOS camera from image collection to FPGA interface. The more detailed picture of the custom-designed CMOS camera is shown in Fig. 2 where the CMOS image sensor and the FPGA4U [20] board is visible. The custom-designed CMOS camera replaces the CCD camera system which includes CCD camera control unit and the CCD camera itself in two separate cases. where the imager inside the camera is Sony-ICX274AL [21]. The case for the camera has two openings from the back and the front where former is for the USB connector and the later is for interfacing output optics of the microscope using a C-mount system. The screw on the left side of the C-mount system is used to adjust coarsely the depth of focus. Although, it is now possible to find CMOS cameras for microscopic applications in the market, at the time of the setting up this system, due to the lack of available CMOS cameras for microscopy, we have chosen to build a custom-designed CMOS camera.

As seen in Fig. 1, the camera system includes an FPGA4u board and a Printed Circuit Board (PCB) specifically built for the CMOS image sensor (MicronMT9V032 [22]). The FPGA4U board includes a USB interface which allows to connect the board to a computer in order to both program the CMOS sensor and transfer the collected images. The collected images are later postprocessed by using MATLAB software on the computer. The image sensor in the camera is a mid-performance black and white CMOS imager with $752 \times 480$ active pixels and 10 bits Analog to Digital Converter (ADC) resolution. The sensor is connected to the FPGA board through the 20 pin connector which carries the Inter-Integrated Circuit $I^{2} C$ bus and the camera control signals. The $I^{2} C$ interface is used to configure the internal registers of the sensor and more specifically the exposure time and analog gain for this application and the Altera Design Software is used to write the VHDL code for camera control and synchronization units and to test them.

A more detailed comparison of the CMOS and CCD camera used in this experiment is shown in Table 1 . The cost of the two cameras given in Table 1 are estimated costs where the CCD camera cost is based on the information in [23] which is an Infinity X32M camera that includes the same sensor as the Nikon CCD camera and the 


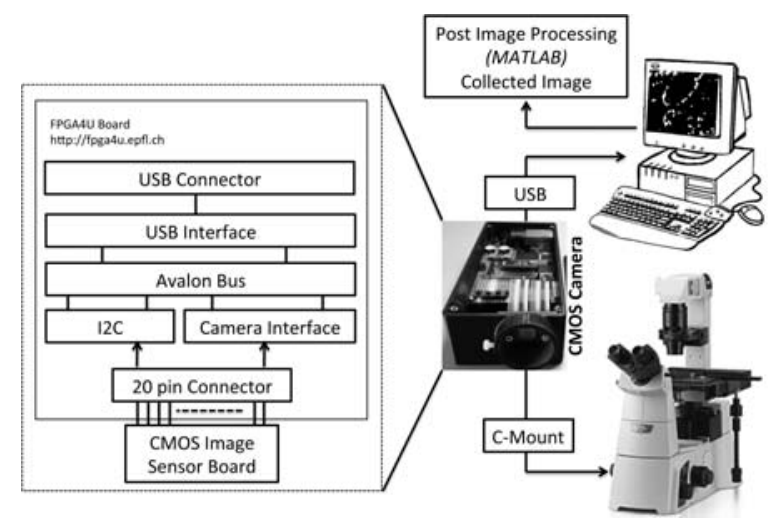

Fig. 1: System Level Representation of Image Collection by CMOS Camera

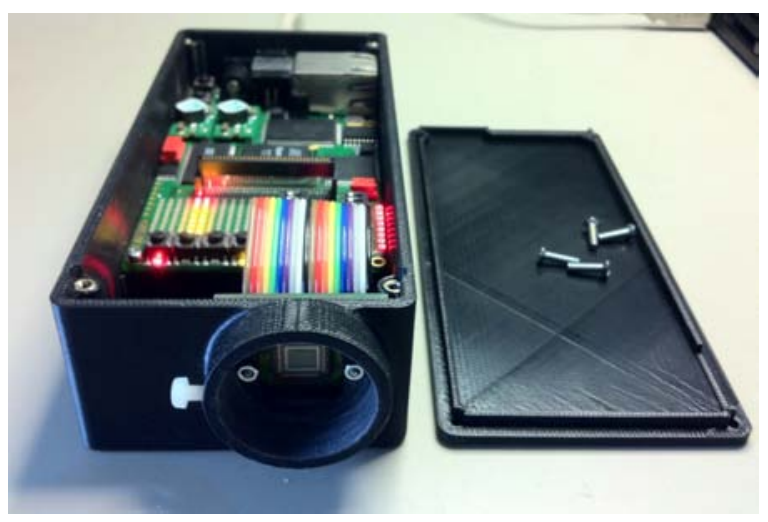

Fig. 2: Custom-Designed CMOS Camera

Table 1: Comparison of CMOS and CCD Cameras Used in this experiment

\begin{tabular}{|c|c|c|}
\hline & Nikon CCD Camera [23] & CD CMOS $[22]$ \\
\hline Sensor & Sony ICX274AL $[21]$ & MT9V032 $[22]$ \\
Optical Format $($ in) & $1 / 1.8$ & $1 / 3$ \\
Pixel Size $(\mu m \times \mu m)$ & $4.40 \times 4.40$ & $6.0 \times 6.0$ \\
Sensor Area $(H \times V)\left(m^{2}\right)$ & 57.8 & 12.99 \\
Number of pixels $(H \times V)$ & $1628 \times 1236$ & $752 \times 480$ \\
Dynamic Range $(d B)$ & 56 & 55 \\
Quantum Efficiency $(Q E) @ 515 n m$ & $\% 54$ & $\% 44$ \\
Pixel Read Noise $\left(e^{-}\right)$ & 12 & 25 \\
Digital Output bits & $8 / 12$ & $\simeq 1600[24]$ \\
Price Euros & 6,300 & \\
\hline
\end{tabular}

CCD Camera

CMOS Camera

CMOS camera cost is based on the Infinity 1-1M CMOS camera [24] whichhassimilarcharacteristicsasthecustomdesigned CMOS camera. The CMOS camera system not only allows a low cost replacement of the CCD camera but also provides a highly flexible and reprogrammable camera unit. It also supports the implementation of additional functionalities and possibly image processing algorithms directly and rapidly on board.

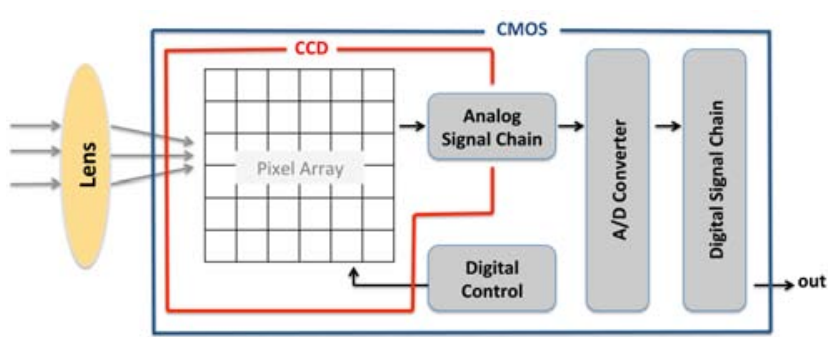

Fig. 3: General View of CMOS and CCD Cameras [25]

Based on the sensor and camera data-sheets, the comparison of the CMOS and CCD cameras used in this experiment are limited to Table 1 . For the CCD camera, since the sensor and camera characteristics vary a lot, the information given on the data-sheet of Infinity X-32M camera is more useful than the image sensor data-sheet but still limited. That is basically because a CCD imager outputs an analog output and consists of pixel array and analog signal chain while a CMOS image sensor consists of pixel array, analog signal chain, on chip noise reduction and digital readout providing a digital output as simply described in Fig. 3 [25]. Thus, the CCD sensor requires extra circuits for noise reduction as well as for digital readout (ADC - Analog to Digital Converter) while a CMOS image sensor generates directly the digital output. The ADC resolution of the CMOS sensor used in this experiment is 10 bits while the extra digital readout circuit combined with the CCD sensor generates 12 bits. For the consistency of the results in this paper, the images collected by the CCD camera is also converted to 10 bits by post-processing. However, it should be notable that it is possible to find CMOS sensors also with 12-bits digital resolution and even higher in the market [26], [27]. The most important parameters that would have a direct impact on the quality of the collected images are the Quantum Efficiency and Read-Out Noise. The read-out noise can further be decreased by post-processing which will be explained in the upcoming sections, however the quantum efficiency $\mathrm{QE}$ which refers to the fraction of photons incident on the detector surface that generate electrons, plays a very important role in the detection limit of the two sensors. As seen in Table 1, the CCD camera has 1.22 times larger QE than the CMOS sensor. However, depending on the application or the light level a lower quantum efficiency can also be enough to generate sufficient number of electrons or for more demanding applications possible to chose CMOS image sensors with higher QEs i.e 77\% at 515nm [27].

To sum up, the comparison made on the sensor performances based on the sensor data-sheets do not provide enough information to draw a conclusion to define the cameras' noise floor or minimum light detection limit 
or their performances for a specific application. The performances given in the camera data-sheets suffer from the same problem as well because of using different terms for the same performance parameters or by not specifying the measurement conditions clearly. Thus, the only comparable information we could achieve from the sensor and camera data-sheets are limited to Table 1. However, the methods presented below can compare the two cameras based on the application-specific collected images which are the images of the artificial and real fluorescent samples. This work mainly compares a widely available high-cost CCD camera for microscopy use with a mid-performance low-cost CMOS camera. Obviously the characteristics of the CMOS sensor can highly be improved by use of better performing sensors from the market [26], [27] or from the literature [28], [29]. For other applications, the same metrics and post-processing algorithms or similar metrics can be used for comparison.

\subsection{Image Intensity Calibration Kit}

A microscope image intensity calibration kit is used to compare the quality of each camera. This kit provides fluorescent microspheres with fluorescenceintensities ranging from very low-intensities, similar to the ones emitted by biological samples, to the brightest signal expected in most microscopy applications. The green calibration kit references as Invitrogen, InSpeck Green (505/515) Microscope Image Intensity Calibration Kit (Life Technologies Incorporation, Carlsbad, CA) used for this application which has Excitation/Emission wavelengths of 505/515 $\mathrm{nm}$ and the diameter of each microsphere is $6 \mu \mathrm{m}$. According to the data sheet of the calibration kit, the kit includes 5 different samples of microspheres at relative intensities of $100 \%, 30 \%, 10 \%, 3 \%, 1 \%$ and $0.3 \%$ which were determined using a Becton Dickinson FACScanTM flow cytometer. However, as listed in the data sheet, the actual relative fluorescence intensities of these components may vary somewhat from the values listed, depending on the kit and the production lot.

\section{METHODS}

In order to provide a cost-efficient solution to high-cost CCDs, standard CMOS camera images are empowered with different image processing algorithms. First of all, Fixed Pattern Noise (FPN)) and temporal noise reduction algorithms are used. Later, we apply thresholding algorithms on CMOS and CCD camera images to extract the morphological patterns on the collected images and to create a comparison metric. In addition, we apply image registration and image resizing algorithms on the images collected by the CCD camera to keep the same area of interest with the images collected by the CMOS camera and we finally compare the camera images from the calculated correlation value. First, we image fluorescence micro-bead samples (size of $6 \mu \mathrm{m}$ ) obtained from microscope intensity calibration kit which emits light at $515 \mathrm{~nm}$ with different Relative Intensities (RIs) as $100 \%$, $33 \%, 10 \%, 3 \%, 1 \%$ and $0.3 \%$. We calculate the RIs of these micro-bead samples by using a new metric called intensity per white pixel (I/WP) and compare the calculated RIs with both cameras. Second, we use a tissue sample obtained from breast cancer patients where an Estrogen Receptor (ER) expression emits low intensity fluorescence light at $665 \mathrm{~nm}$. More details on the tissue sample imaging can be found in [30]. Finally, we also applied the methods on images of fluorescent groups of Caco-2 cells. Using immunofluorescence techniques, we stained the naturally present Glyceraldehyde 3-phosphate dehydrogenase (GAPDH) enzyme that we use as an example of nanometer-scale target using a Texas-red compatible dye.

\subsection{Image Processing Algorithms Applied on the CMOS Camera Images}

\subsubsection{Noise Removal}

The goal of this research is to deal with low light emitting samples and applications. This is why noise contributors should be dealt with by reducing their impact on the useful signals. CMOS imagers are known to suffer from various noise sources which can be classified either as temporal noise or FPN [31]. Temporal noise (e.g. photon shot noise, readout noise) results from a stochastic process and cannot be fully determined nor mitigated for every pixel. However, the FPN is not a function of time and can be determined. It forms a constant pattern among the pixels/columns of the imager sensor. This problem arises from small differences in the individual responsivity of the pixels or the column amplifiers that are mostly caused by inhomogeneity in the manufacturing process. The noise removal algorithms are depicted in the results section.

\section{Fixed Pattern and Temporal Noise Reduction}

The FPN is generally divided into two components; dark signal non-uniformity (DSNU) and pixel response nonuniformity (PRNU). DSNU is an offset between pixels in dark without illumination (dark current generation variability) and PRNU is seen as a responsivity variation among pixels under illumination. Both of these noise sources are affected by the exposure time, the imager temperature and the imager analog gain. When the light intensity received by the CMOS sensor through the fluorescence microscope is weak, it is required to program 
the CMOS image sensor at high exposure time and analog gain. This causes a huge FPN to appear and a classic method to mitigate part of the DSNU is applied [32] on the CMOS images. First of all, a master dark frame (MDF) is generated by computing the median or the average frame out of a set of $N$ dark frames. Second, the MDF is subtracted from any regular captured bright frame (i.e. containing the signal) at the same exposure and gain as the MDF. The de-noised frames are computed using;

$F_{\text {corrected }}=\max \left(0, F_{\text {raw }}-M D F\right)$.

The sensor temperature should be stable during the calibration process, which can be achieved by letting the system on for a few minutes before capturing the dark frames.

On the other side, temporal noise is a function of time and includes different noise sources such as photon shot noise and readout noise. By collecting multiple images and averaging the collected images, temporal noise has been reduced.

\section{Removal of Hot Spots/Pixels and Dead Pixels} In every CMOS and CCD camera, there are dead and hot pixels. The amount of these defective pixels depends on temperature, technology, design, layout or microlenses. They may also appear due to aging of the sensor. Hot pixels generate higher leakage or dark current than normal. When an image is taken under long-exposure time, longer than causing the pixel exceeding its linear charge capacity, they appear as bright spots and cause salt and pepper type noise on the image. This type of noise cannot be removed by MDF generation and subtraction, since it is only visible at high exposure time. On the other hand, dead pixels are unresponsive stuck pixels and no matter what the light intensity or exposure time, they do not respond to light. A common method to remove hot pixels or dead pixels is replacing them by the median value of the surrounding pixels. This remapping operation can be done by MATLAB median filtering - medfilt2 operand or by an outlier removal algorithm. For this experiment, the following outlier removal algorithm has been used;

$\Delta=\left(\left|I_{i j}-m\right|\right)$

$\forall i \in[1, v], \forall j \in[1, h]: I_{i, j}=\left\{\begin{array}{l}m \text { if } \Delta>T h, \\ I_{i j} \text { else. }\end{array}\right.$

$I$ : Intensity value of a pixel

$h$ : Number of pixels at the horizontal direction $v$ : Number of pixels at the vertical direction $m$ : Median value of the intensity values of pixels in a certain window around the chosen pixel $I_{i j}$ window: Defined array size (radius $\times$ radius $)$.

If the $\Delta$ value is above a defined Threshold $(T h)$, the intensity value of the chosen pixel $\left(I_{i j}\right)$ is replaced by the calculated median $(m)$ and else if the $\Delta$ is below the $T h$, no change is done and the pixel value is kept as it is. This method can be applied for both hot and dead pixels. Hot pixels are the pixels that exceed the level of the brightest neighboring pixel by more than the $T h$ and the dead pixels are the pixels that are darker than the darkest neighboring pixel by more than the $T h$. In both cases, they are replaced by the median of the surrounding pixels.

\subsubsection{Auto-Thresholding}

Thresholding method aims at selecting a threshold by maximizing a criterion measure that evaluates the "goodness" of that threshold. For the experiments that are stated in this paper, automatic thresholding method introduced by N.Otsu [33] is applied on the images by using MATLAB's graythresh function.

During the thresholding process, individual pixels in an image with an intensity value larger than a defined threshold value are converted to 1 ( "object" pixels) where all theother pixelvalues below this threshold areconverted to 0 ( "background" pixels). Otsu's thresholding is a nonparametric method automatically selecting a threshold level for a gray-level image based on its histogram. The algorithmconsiders theimagetobethresholded consisting of two classes of pixels as foreground and background and tries to achieve a thresholding value which minimizes the intra-class variation while at the same time allowing the maximization of the inter-class variation.

The only input of the method is the normalized graylevel histogram of the image, which can also been seen as a probability distribution. Given a threshold value, the $L$ bins of the histogram can be dichotomized in two classes; $C_{0}$ gathering the bins indexed by $[0, . ., k-1]$ and $C_{1}$ gathering the bins indexed by $[k, . ., L-1]$. The gray level corresponding the bin $k$ corresponds to the selected threshold.

Finding the optimal threshold $k^{*}$ is reduced to solving

$\max _{S^{*}}\left(\sigma_{B}^{2}(k)\right)$

where $S^{*}$ is the range of $k$ over which the maximum is sought

$S^{*}=\left\{k ; \omega_{0} \omega_{1}>0\right.$, or, $\left.0<\omega_{0}<1\right\}$

and $\sigma_{B}^{2}$ is referred to as the between-class variance defined by

$\sigma_{B}^{2}=\omega_{0} \omega_{1}\left(\mu_{1}-\mu_{0}\right)^{2}$ 
for which $\omega_{0}$ and $\omega_{1}$ are the probability of class occurrences and $\mu_{0}$ and $\mu_{1}$ and the class mean levels.

\subsubsection{Image Registration}

When the camera sensors have different pixel and pixel array sizes, image registration algorithms should be applied on the collected images to reach a fair comparison. Image registration is the process of aligning the pixels of two or more images of the same scene when one image is considered as a reference. In this experiment, the image registration algorithms basically include rotation, cropping and scaling of the CCD camera images according to the CMOS camera images since the CCD array size is larger than the CMOS one. Below are the steps used for the registration of the CCD camera images until highest correlation with the CMOS camera image is achieved:

1) Rotation of the image to solve the low or high angles of tilt issues that may appear when mounting the cameras (with MATLAB's imrotate function).

2) Cropping of the CCD camera image to reach same area of interest with the CMOS camera image. In this experiment, MATLAB's imcrop function is used to crop the CCD camera image of $1628 \times 1236$ according to the field of view of the CMOS camera image and finally an image with an array size of $989 \times 631$ is reached.

3) Resizing/Scaling of the CCD camera image. The scaling factor for horizontal and vertical directions should be calculated separately depending on the size of the each camera pixel. In this experiment, both of the camera pixels are in square which results in the same horizontal and vertical scaling factors. The scaling factor is calculated by dividing the CMOS pixel size to the CCD pixel size which is 1.3159 (CMOS pixel size / CCD pixel size = $5.79 \mu \mathrm{m} / 4.4 \mu \mathrm{m}=1.3159)$. By using the scaling factor, the cropped CCD image of $989 \times 631$ is resized to an image with an array size of $752 \times 480$.

\subsection{Calculation of the Relative Intensities from the Calibration Kit Samples}

For measuring the microsphere RIs, we mount each microsphere sample of $5 \mu \mathrm{L}$ on a separate glass slide. After letting each droplet dry on the glass slide, we imaged the samples with both CCD and CMOS cameras at different Neutral Density (ND) filter values where an ideal ND filter modifies the intensity of light equally according to its value. The collected images are later used to calculate the relative intensity values at the correct ND level as a comparison method. However, it is not straightforward to calculate the relative intensities of each sample since the number of microspheres per droplet is not known and their distribution on the glass slide is not uniform which causes a varying number of microspheres for each sample and for each frame. This is why a metric called intensity per white pixel (I/WP) has been developed by using the calculated parameters total intensity (TI) and White pixel (WP). It is important to pay attention to the different pixel array size of CMOS and CCD sensors when annotating these parameter values. The CMOS sensor has $752 \times 480$ pixels while the CCD sensor has $1628 \times 1236$. Ideally, this would result in 5.57 times larger TI and WP in CCD images than CMOS ones if the responsivity and noise level of two sensors were the same and micro-spheres have been uniformly distributed. However, none of these conditions are valid and the number of microspheres per image and its ratio to the dark areas somehow differ from an ideal distribution. Thus, it is expected to achieve larger values of TI and WP for CCD images than CMOS ones but it is not possible to define the exact ratio of this increase. On the other hand, the I/WP and RI parameters are independent of the area that is imaged or the pixel array size of the camera that is used, or the non-uniform distribution of the micro-beads. The immunity of these parameters to different conditions make these parameters reliable for this application and they are also easily reusable for any camera comparison. The RI parameter calculated from the I/WP parameter is used as a comparison metric for this application since the micro-beads RI values are already known within a margin. Details of the calculations for each parameter is given below.

The total intensity (TI) parameter is the sum of the each pixel intensity values on a grayscale image and calculated as;

$I_{\text {Total }}=\sum_{i=1}^{v} \sum_{j=1}^{h} I_{i j}$

I: Intensity value of a pixel

h: Number of pixels at the horizontal direction

$\mathrm{v}$ : Number of pixels at the vertical direction

White pixel (WP) parameter is related with the thresholding concept which is explained earlier. By summing up the pixels above the threshold value, the total number of white pixels (WP) in an image can be calculated.

$\forall i \in[1, v], \forall j \in[1, h]: B W_{i, j}=\left\{\begin{array}{l}0 \text { if } I_{i j}<V_{\text {Thresholding }} \\ 1 \text { if } I_{i j} \geq V_{\text {Thresholding }}\end{array}\right.$

where $V_{\text {Thresholding }}$ is the thresholding value calculated by MATLAB and BW is the pixel value after thresholding, 
either $\operatorname{black}(0)$ or white(1).

$B W_{\text {Total }}=\sum_{i=1}^{v} \sum_{j=1}^{h} B W_{i j}$

By dividing the total intensity (TI) in a grayscale image to the total number of WPs, the intensity per white pixel (I/WP) parameter is defined.

$I / W P=\frac{I_{\text {Total }}}{B W_{\text {Total }}}$

In order to improve the reliability of the RI results, the I/WP parameter is averaged over twenty images of the same sample which can be the depicted as $\mu_{N}$. The RI value among different samples can be formulated by below equation where $\mathrm{X}$ represents the imaged sample that the RI is being calculated of.

$R I=\frac{\sum_{N=1}^{20} \mu_{N}(\text { Sample } \% X)}{\sum_{N=1}^{20} \mu_{N}(\text { Sample } \% 100)}$

\section{RESULTS AND DISCUSSION}

\subsection{Comparison on Relative Intensity}

Table 2: Relative Intensity Calculations from CMOS Camera Images of Microsphere Slot of Relative Intensity $10 \%$

\begin{tabular}{cccc}
\hline \hline $\mathrm{ND}$ & $\sum($ Intensity $)$ & $\sum($ WhitePixel $)$ & TI/WP x ND \\
\hline 1024 & 1253 & 115229 & 11.1370 \\
512 & 1279 & 116210 & 5.6347 \\
256 & 1437 & 122685 & 2.9993 \\
128 & 1739 & 19519 & 11.9592 \\
64 & 2219 & 11096 & 12.7979 \\
32 & 3514 & 11201 & 10.0401 \\
16 & 5483 & 11158 & 7.8616 \\
8 & 9808 & 11236 & 6.9832 \\
4 & 15826 & 12312 & 5.1418 \\
2 & 20996 & 14812 & 2.8349 \\
1 & 30328 & 19845 & 1.5282 \\
\hline
\end{tabular}

In Table 2 and 3 , sum of intensities of gray scaleimagesTI, sum of number of white pixels after thresholding - WP, and intensity over white pixel - I/WP results are shown for the micro-beads with $10 \%$ of relative intensity with different ND values so the illumination levels. In Fig. 4, the sum of intensity values on Table 2 and 3 are graphically represented and it is seen that for ND values from 1 to 4, the pixels are saturated and from 128 to 1024 they are under-illuminated. ND values from 8 to 64 represent an area of interest where WP count is almost constant
Table 3: Relative Intensity Calculations from CCD Camera Images of Microsphere Slot of Relative Intensity $10 \%$

\begin{tabular}{cccc}
\hline \hline CMOS & CCD & & \\
\hline ND & N(Intensity $)$ & $\sum($ WhitePixel $)$ & TI/WP x ND \\
\hline 1024 & 12404 & 611848 & 20.7872 \\
512 & 12971 & 632442 & 10.4196 \\
256 & 14127 & 653572 & 5.5296 \\
128 & 16759 & 62725 & 34.2016 \\
64 & 20852 & 57960 & 23.0272 \\
32 & 30819 & 57511 & 17.1488 \\
16 & 46833 & 57213 & 13.0976 \\
8 & 76800 & 59319 & 10.3576 \\
4 & 101165 & 68321 & 5.9056 \\
2 & 134034 & 84263 & 3.1814 \\
1 & 195104 & 114228 & 1.7080 \\
\hline
\end{tabular}

as expected among light power. The WP parameter can also be used to define the exact illumination level. When decreasing the illumination level from the highest to the lowest by using the ND filters (changed from 1 to 1024), it is found that the WP first starts decreasing due to the decrease in the number of saturated pixels, and after some point, this value start re-increasing due to the noise falling into the threshold level. When noise falls into the threshold level, the noise is also counted as part of the morphological pattern and the black and white image is no longer correct. This is why the ND value corresponding to the lowest WP is considered as the correct illumination level for the sake of this measurement and highlighted in yellow in Table 2 and 3. This behaviour could be observed in all samples at different RIs although in here it is shown only for fluorescence micro-beads with illumination levels of $\% 10$.

The TI and WP parameters for CMOS and CCD images largely differ due to the large array size of the CCD sensor, the non-uniform distribution of the microbeads, the unknown ratio between white and black pixels and the difference in performance parameters of the two sensors. TheWP parameterislessimmunetothedifference in the performance parameters but still immune to nonuniformity and to the unknown ratio. The results of this parameter differ by a ratio of 5.2 for the two cameras which is close to the ideal value (CCD array size / CMOS array size $=5.57)$. Thus, neither the TI nor the WP parameters can be used to calculate the RIs. As mentioned earlier, the $\mathrm{I} / \mathrm{WP}$ parameter is a better comparison parameter since it is not dependent on the array size or the non-uniformity of micro-beads' distribution. The highlighted rows of Table 2 and 3 show that the calculated I/WP for the CCD image is 13.09 and 7.86 for the CMOS camera image for the same illumination level. This value can be interpreted similar to the system gain $(K)$ in the linear region of a sensor which is defined as DigitalNumber $(D N) / e^{-}$. 
This commonly known system gain parameter defines the number of digital numbers per electron or vice versa, where in this method the I/WP parameter defines the number of digital numbers per white pixel.

The relative intensity - RI parameter on the other side compares the two cameras in terms of their imaging capabilities providing a relative value with respect to the highest intensity sample. Thus, we achieve a direct comparison of the sensors imaging capabilities for this light range. The calculation method for RIs and results are shown in the next section.

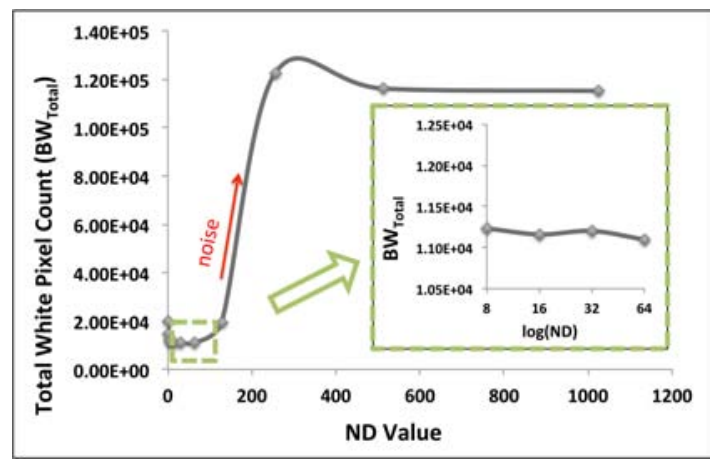

(a) Measured on Images Collected by CMOS Camera

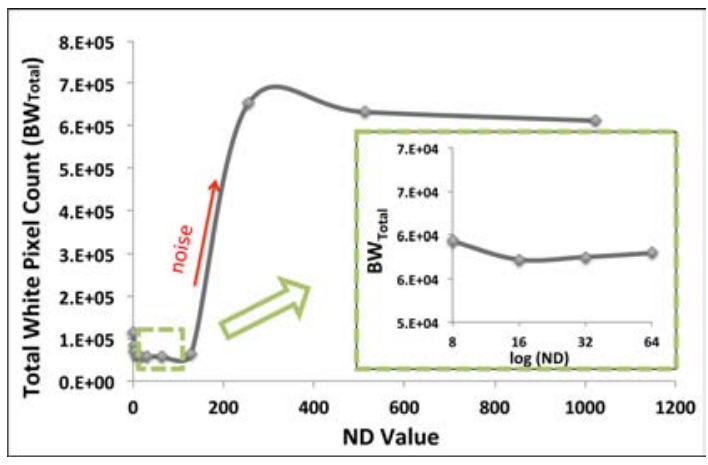

(b) Measured on Images Collected by CCD Camera

Fig. 4: Total white pixel Variation of a Thresholded Image with respect to ND Filter Values

Table 4: Calculated Relative Intensities of Microspheres by CMOS Camera

\begin{tabular}{ccccc}
\hline \hline Expected RI & $\mu_{N}$ & $\mathrm{ND}$ & $\mu_{N} \times N D$ & Calculated RI \\
\hline$\% 100$ & 0.4222 & 256 & 108.1 & $\% 100$ \\
$\% 30$ & 0.3559 & 128 & 45.55 & $\% 42.15$ \\
$\% 10$ & 0.4914 & 16 & 7.86 & $\% 7.27$ \\
$\% 3$ & 0.3458 & 8 & 2.77 & $\% 2.56$ \\
$\% 1$ & 0.5657 & 2 & 1.13 & $\% 1.05$ \\
$\% 0.3$ & 0.5072 & 1 & 0.51 & $\% 0.47$ \\
\hline
\end{tabular}

Table 5: Calculated Relative Intensities of Microspheres by CCD Camera

\begin{tabular}{ccccc}
\hline \hline Expected RI & $\mu_{N}$ & $\mathrm{ND}$ & $\mu_{N} \times N D$ & Calculated RI \\
\hline$\% 100$ & 0.76 & 256 & 194.81 & $\% 100$ \\
$\% 30$ & 0.6053 & 128 & 77.48 & $\% 39.77$ \\
$\% 10$ & 0.82 & 16 & 13.10 & $\% 6.72$ \\
$\% 3$ & 0.6065 & 8 & 4.85 & $\% 2.49$ \\
$\% 1$ & 0.55 & 4 & 2.2 & $\% 1.13$ \\
$\% 0.3$ & 0.91 & 1 & 0.91 & $\% 0.47$ \\
\hline
\end{tabular}

\subsection{Comparison on Imaging}

In Table 4 and 5 , the calculated RIs for both CMOS and CCD camera images are shown with the parameters that are used. The calculated results vary from the expected relative intensities for both CMOS and CCD camera images. The variation for CMOS images for all intensity levels in average is $28.6 \%$ while it is $30.4 \%$ for the CCD images. This variation was expected as stated from the data-sheet of the fluorescence due to the variation of the production lot and also due to the difference in the calculation technique. However, despite the variation of calculated RIs from the expected values, the results are consistent for both CMOS and CCD camera images and the variation among the two camera images is only $\% 4.79$. This means that both cameras are capable of generating similar quality images as well as close quantitative results from a large-scale intensity fluorescence samples.

In this section, the image processing algorithms explained in Section 3.1 are applied on the CMOS and/or CCD camera images step by step. First, due to the high exposure time in CMOS camera, FPN noise becomes critical and FPN noise reduction algorithm is applied on both Tissue and Caco-2 cells' CMOS camera images. Second, to remove both hot and dead pixel outliers which are numerically distant from the surrounding pixel values on the image, outlier removal algorithm is applied on the CMOS and CCD camera images. Later, autothresholding algorithm is used for both camera images for quantitative calculations and comparison as well as for better visibility of the morphological patterns expressed on the cells. Finally, the CCD camera images are registered and resized according to the CMOS camera images. With this method, the CCD images of $1200 \mathrm{Vx} 1600 \mathrm{H}$ are converted to images of $480 \mathrm{Vx} 752 \mathrm{H}$. In order to keep the experimental setup the same for both cameras, the same light intensity (ND Filter $=1$ ) and microscope optics and objectives (40X, Numerical Aperture $=0.75$ ) are used for both camera image acquisitions. The light emitted from 


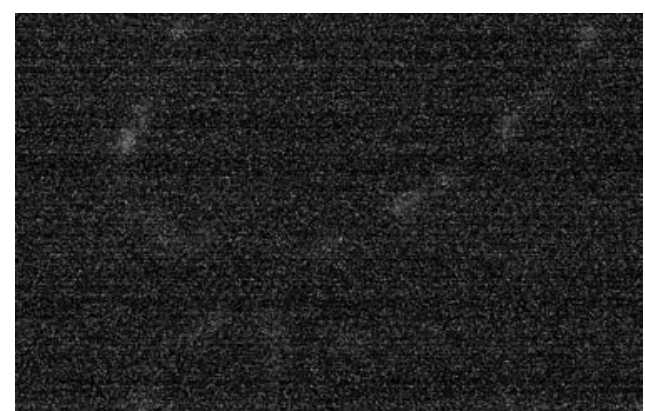

(a) CMOS Gray-Scale Camera Image Before Noise Removal (with DSNU)

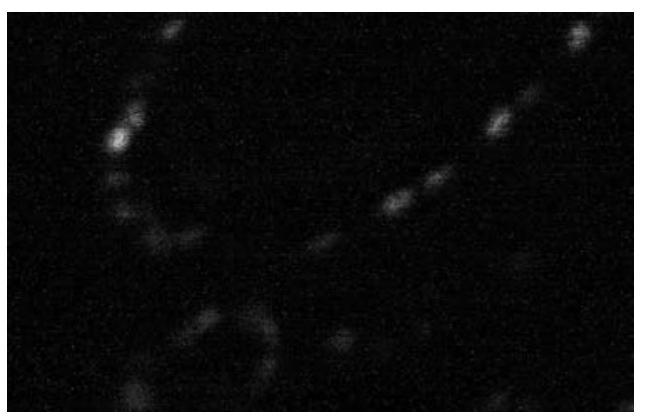

(c) CMOS Gray-Scale Camera Image After FPN Removal (without DSNU) (Contrast Enhancement for Better Display of Salt and Pepper Noise Caused by Hot Pixels

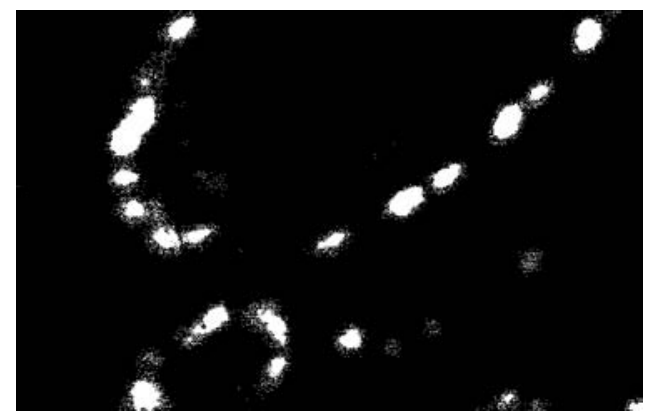

(e) CMOS Camera B/W Image After Otsu's Auto-Thresholding Method

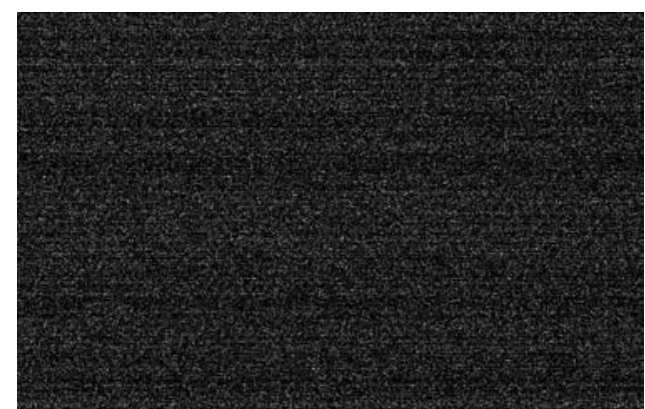

(b) CMOS Camera Master Dark Frame (MDF)

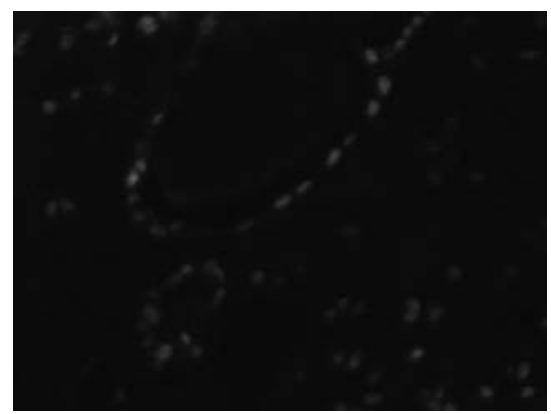

(d) CCD Camera Gray-Scale Image (Enhanced Contrast)

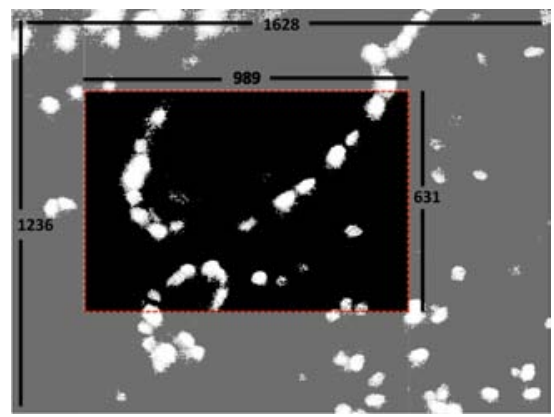

(f) CCD Camera BW Image after Otsu's Auto-Thresholding Method

Fig. 5: CMOS and CCD Camera Images of ER detection from Tissue Samples (Exposure=1s, Gain=8x) [30].

theER expression in tissue cells and the Gabdh expression in Caco-2 cells are both much lower than the micro-beads even at lowest intensities. That is why the samples have been imaged at very high exposure time (1s) and analog gain $(8 \mathrm{X} / 16 \mathrm{X})$ for both camera experiments.

\subsubsection{Tissue Sample Imaging}

The sample is a breast cancer diagnostic sample that the nuclear Estrogen Reception (ER) expression emits fluorescence light at $665 \mathrm{~nm}$. ER is detected by indirect immunohistochemicalreaction [34], [35] using monoclonal mouse anti-human anti-ER receptor antibody as primary antibody (clone 6F11, Leica Microsystems) and AlexaFluor 647 conjugated goat anti-mouse polyclonal IgG antibody (Invitrogen) as secondary anti-body.

First, from the CMOS camera row images (Fig. 5a), the Master Dark Frame (MDF) (Fig. 5b) is subtracted and the corrected image is obtained as seen in Fig. 5c. Contrast enhancement is applied on this image in order to increase the visibility of hot pixels/spots. Later, outlier removal algorithm is applied on this image. Finally, thresholding method is applied on the image which is shown in Fig. 5e which improves the localization of the morphological pattern. For the CCD images seen in Fig. $5 \mathrm{~d}$, again outlier removal algorithm is applied as well 


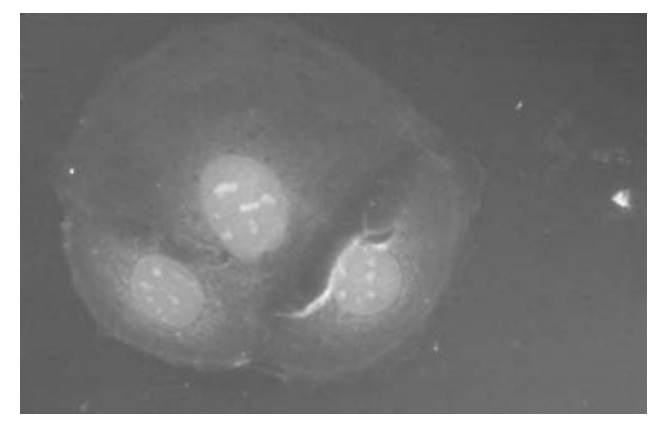

(a) CMOS Gray-Scale Camera Image After FPN Removal (without DSNU)

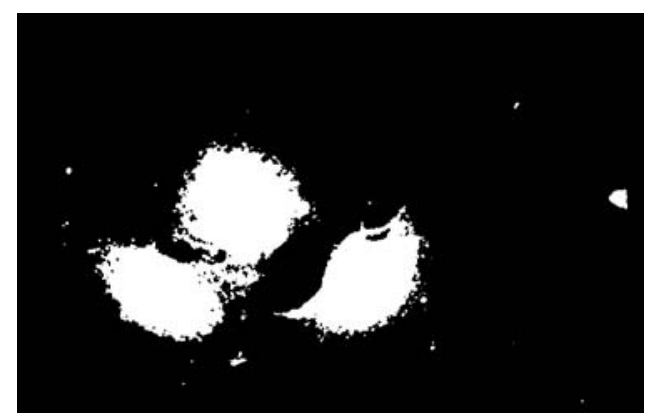

(c) CMOS Black and White Camera Image After Otsu's Auto-Thresholding Method

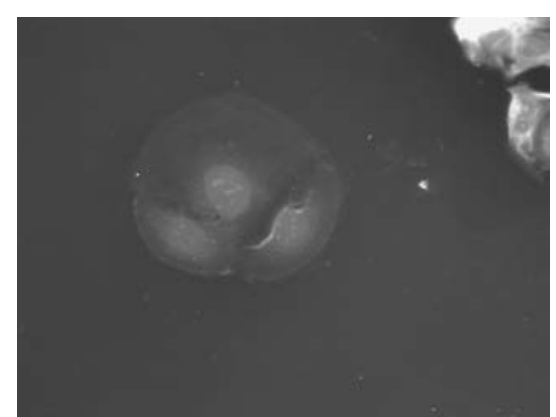

(b) CCD Gray-Scale Camera Image

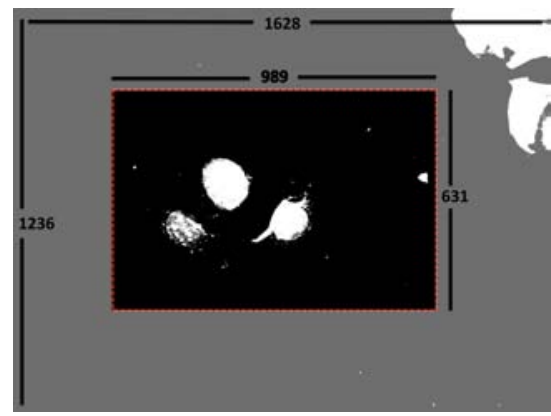

(d) CCD Black and White Camera Image After Otsu's Auto-Thresholding Method

Fig. 6: CMOS and CCD Camera Images of Gabdh Protein Expression on Caco-2 Cells (Exposure=1s, Gain=16x)

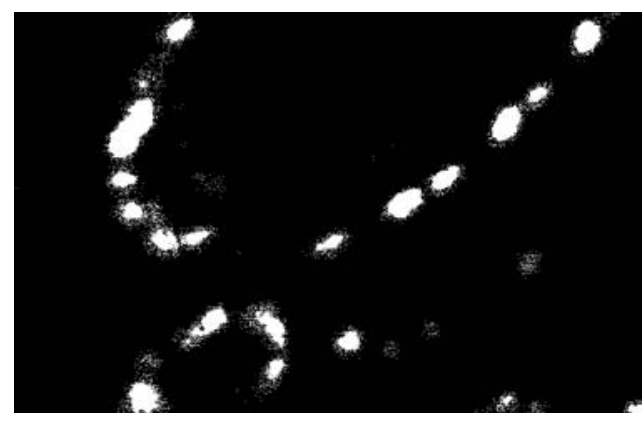

(a) Tissue Sample Image with CMOS camera

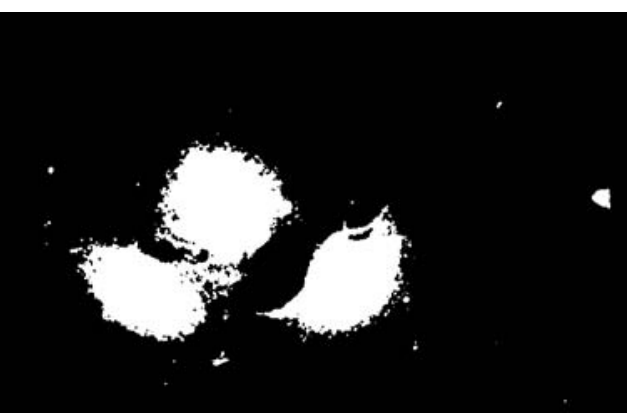

(c) Caco-2 Cell Line CMOS Camera Image

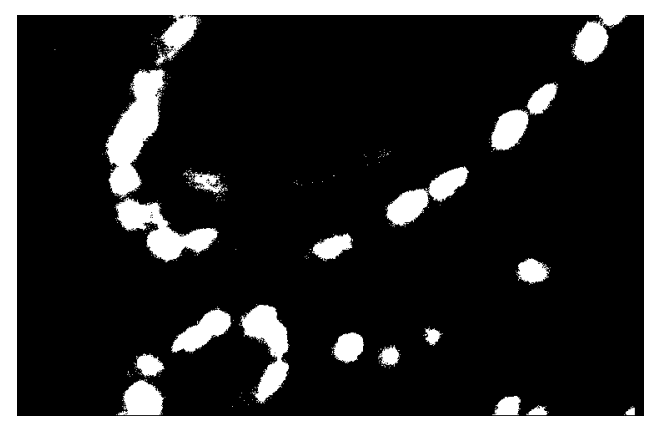

(b) Registered and Resized CCD Camera Tissue Sample Image $(752 \times 480)$

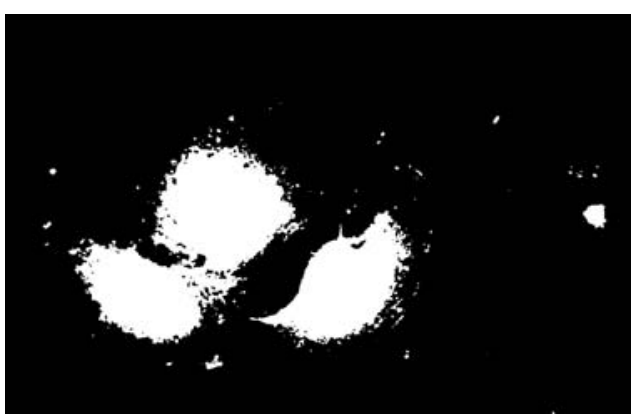

(d) Registered and Resized CCD Camera Caco-2 Cell Line Image $(752 \times 480)$

Fig. 7: CMOS and CCD Camera Image Comparison 
as the auto-thresholding method. Resulting image after auto-thresholding is seen in Fig.5f.

\subsubsection{Gabdh Gene Expression on Caco-2 Cells}

Human colon adenocarcinoma (Caco-2) cells are commonly used in pharmaceutical researches as an in-vitro model of the human small intestinal mucosa in order to monitor the drug uptake and transport. Since the culturing of mature intestinal epithelial cells are very difficult, recently Caco-2 cell lines have taken a lot of attention. In [36], Caco-2 cell monolayers have been proposed as a model for drug transport across the intestinal mucosa. In addition, in [37], induction of Toll-like receptor - TLR proteins with Lipopolysaccharides - LPS has been shown on Caco-2 cells to investigate the inflammation in Gastro-intestinal Tract - GIT epithelial cells and a nutrition platform mimicking the human GIT within the frame of these results have been proposed.

Due tothepopularity ofCaco-2 cellsin pharmaceutical research and nutrition analysis on human health, we use a Caco-2 cell sample with Gabdh protein expression to show the possible use of CMOS cameras on these kind of applications.

Similar to the tissue sample images, again CMOS camera images are corrected with FPN removal algorithm and converted to black and white images with autothresholding algorithm as seen in Figures $6 \mathrm{a}$ and $6 \mathrm{c}$. For the CCD camera Caco-2 cell images, the same procedure as in the tissue sample imaging is applied and results are shown in Figures $6 \mathrm{~b}$ and $6 \mathrm{~d}$. Three Caco-2 Cells are visible in these images and the Gabdh protein is expressed in the nucleus of the cells.

\subsubsection{Comparison of CCD and CMOS camera images}

Fig. 7 helps to make a direct comparison on the CMOS and registered and resized CCD camera tissue and Caco-2 cell images.

In Figures, $7 \mathrm{a}$ and $7 \mathrm{~b}$, it is seen that both cameras are capable of detecting the morphological pattern of the ER receptor expression at the nucleus level that can be found in the breast tissue. The correlation coefficient calculated among these two images by using the MATLAB cross-correlation function of corr2 is found to be 0.65 .

In Figures, $7 \mathrm{c}$ and $7 \mathrm{~d}$, it is visibly possible to conclude that both cameras are capable of detecting the morphological pattern of the Gabdh protein expression at the Caco-2 cell nucleus level. A correlation value among these two black and white images is found as 0.84 .

These high correlation values confirm the conclusion that was drawn earlier that the CMOS cameras when empowered with image processing algorithms should be considered for cellular level optical studies.

\section{CONCLUSION}

In this paper we proposed a comparative study between a CCD and a CMOS camera with respect to their performances for imaging on artificial fluorescence beads and a biological tissue sample by using optical microscopy. The calculations applied on theimages of artificial fluorescence beads have shown that even a mid-performance, low-cost CMOS and a high-cost CCD cameras extract very close information where the final variation among the relative intensities is only $4.79 \%$. For the cancer diagnostic and Caco-2 samples, since ER and Gabdh expressions emit even lower fluorescence light than the micro-beads with the lowest intensities, the effect of the noise reduction algorithms have become even more crucial and visible. At this high exposure rates, although the initial CMOS image has been very noisy, after applying proper imageprocessing algorithms, the CMOS camera was capable of generating the same morphological pattern as the CCD camera image. Therefore, this paper demonstrates that CMOS cameras are recommended for investigations of cells and tissues when dealing with fluorescence microscopy. It paves the way to biologists to further investigate their camera options as well as decrease their instrument costs. Undoubtedly, the trend towards using low-cost CMOS cameras is even more important when standard microscopy is replaced by (possibly disposable) lab-on-chip platforms.

\section{Acknowledgment}

The research work presented in this paper was funded by the NutriCHIP project with a grant from the Swiss Nano-Tera.ch initiative, evaluated by the Swiss National Science Foundation. It was also partially supported by the NanoSys project, the program ERC-2009-AdG-246810. Finally, the authors would like to thank to A ta Tuna Çiftlik fromLMIS2 (MicrosystemsLaboratory 2), EPFL for their support in tissuesamplepreparation and Ali Galip Bayrak from LAP (Processor Architecture Laboratory), EPFL for his precious suggestions and the useful discussions.

\section{References}

1. Fossum, E. R., Hynecek, J., Tower, J., Teranishi, N., Nakamura, J., Magnan, P., \& Theuwissen, A. (2009). "Special Issue on Solid-State Image Sensors". Electron Devices, IEEE Transactions on, 56(11), 2376-2379.

2. Ansorge, W. J. (2009). "Next-generation DNA sequencing techniques". New biotechnology, 25(4), 195-203. 
3. Sapuppo, F., Intaglietta, M., \& Bucolo, M. (2008). "Biomicrofluidics real-time monitoring using CNN technology". Biomedical Circuits and Systems, IEEE Transactions on, $2(2), 78-87$.

4. Kang, H. W., Muramatsu, H., Lee, B. J., \& Kwon, Y. S. (2010). "Monitoring of anticancer effect of cisplatin and 5 -fluorouracil on HepG2 cells by quartz crystal microbalance and micro CCD camera". Biosensors and Bioelectronics, 26(4), 1576-1581.

5. Osmani, N., Peglion, F., Chavrier, P., \& Etienne-Manneville, S. (2010). "Cdc42 localization and cell polarity depend on membrane traffic". The Journal of Cell Biology, 191(7), 1261-1269.

6. Nicolini, C., Carrara, S., \& Mascetti, G. (1997). "High order DNA structure as inferred by optical fluorimetry and scanning calorimetry". Molecular biology reports, 24(4), 235-246

7. Mascetti, G., Vergani, L., Diaspro, A., Carrara, S., Radicchi, G., \& Nicolini, C. (1998). "Effect of fixatives on calf thymocytes chromatin as analyzed by 3D high-resolution fluorescence microscopy". Cytometry, 23(2), 110-119.

8. Mascetti, G., Carrara, S., \& Vergani,L. (2001). "Relationship between chromatin compactness and dye uptake for in situ chromatin stained with DAPI". Cytometry, 44(2), 113-119.

9. Fossum, E.R. (1993), "Active pixel sensors: Are CCD's dinosours?". Proc. SPIE, vol. 1900, pp. 1-3.

10. Fossum, E.R. (1997), "CMOS image sensors: Electronic camera-on-chip", IEEE Trans. Electron Devices, vol. 44, pp. 1689-1698.

11. Murari, K., Etienne-Cummings, R., Cauwenberghs, G., \& Thakor, N. (2010, August). "An integrated imaging microscope for untethered cortical imaging in freely-moving animals". In Engineering in Medicine and Biology Society $(E M B C), 2010$ Annual International Conference of the IEEE (pp. 5795-5798).

12. Murari, K., Greenwald, E., Etienne-Cummings, R., Cauwenberghs, G., \& Thakor, N. (2009, September). "Design and characterization of a miniaturized epi-illuminated microscope". In Engineering in Medicine and Biology Society, 2009. EMBC 2009. Annual International Conference of the IEEE (pp. 5369-5372).

13. Ghosh, K. K., Burns, L. D., Cocker, E. D., Nimmerjahn, A., Ziv, Y., El Gamal, A., \& Schnitzer, M. J. (2011). "Miniaturized integration of a fluorescence microscope". Nature methods, 8(10), 871-878.

14. Li, D. U., Arlt, J., Richardson, J., Walker, R., Buts, A., Stoppa, D., ... \& Henderson, R. (2010). "Real-time fluorescence lifetime imaging system with a $32 \times 320.13 \mu \mathrm{m}$ CMOS low dark-count single-photon avalanchediodearray". Optics express, 18(10), 10257-10269.

15. Schwartz, D. E., Charbon, E., \& Shepard, K. L. (2008). "A single-photon avalanche diode array for fluorescence lifetime imaging microscopy". Solid-State Circuits, IEEE Journal of, 43(11), 2546-2557.

16. Mutch, Sarah A., et al. "Deconvolving single-molecule intensity distributions for quantitative microscopy measurements." Biophysical journal 92.8 (2007): 2926-2943.

17. Cronin, Brd, Ben de Wet, and Mark I. Wallace. "Lucky imaging: Improved localization accuracy for single molecule imaging." Biophysical journal 96.7 (2009): 2912-2917.

18. J. Ghaye, M. A. Kamat, L. Corbino-Giunta, P. Silacci, G. Vergeres, G. De Micheli, S. Carrara (2013). "Image Thresholding Techniques for Localization of Sub-Resolution Fluorescent Biomarkers". Cytometry part a, in press.

19. Ghaye, Julien, Giovanni De Micheli, and Sandro Carrara. "Simulated biological cells for receptor counting in fluorescence imaging." BioNanoScience 2.2 (2012): 94-103.
20. Favi, C., Beuchat, R., Jimenez, X., \& Ienne, P. (2009, October). "From gates to multi-processors learning systems hands-on with FPGA4U in a computer science programme". In Proc. of the 2009 Workshop on Embed. Sys. Edu.(WESS), Grenoble, France.

21. PointGrey Research (Richmond, BC, Canada) (Jul, 2010), Technical Application Note TA N2008006, availableat http://www.ptgrey.com/support/downloads/documents_/TAN2008006_S Accessed 2 Jul, 2012.

22. Aptina Imaging Corporation, San Jose, CA, 1/3-Inch Wide-VGA CMOS Digital Image Sensor, available at http://www .aptina.com/products/image_sensors/mt9v032d00stm/. Accessed 2 Jul, 2012.

23. Lumenera Corporation, Infinity X-32M, 32 Megapixel CCD USB 2.0 Camera, available at http://www.emsdiasum. com/microscopy/technical/datas_heet/95116_ Accessed 2 Jul, 2012.

24. Lumenera Corporation, Infinity 1-1M, 1.3 Megapixel monochrome camera, available at http://www.oem-optical.com/lumenera-cmos-1model.html. Accessed 2 Jul, 2012.

25. Theuwissen, A. "Digital Imaging: Image Capturing, Image Sensors - Technologies and Applications". Annual International Courses in Telecommunications Semiconductor TechnologyNanotechnology, CEI-Europe, Barcelona-Spain, 2012.

26. Aptina Imaging Corporation, San Jose, CA, 1/2.5Inch 5MP CMOS Digital Image Sensor, available at http://www .aptina.com/products/image_sensors/mt9p031i12stm/. Accessed 2 Jul, 2012.

27. Aptina Imaging Corporation, San Jose, CA, 1 Megapixel 1/3-Inch Digital Image Sensor Recon and $i L C C$, available at http://www.aptina.com/products/image_sensors/ar0130cs. Accessed 2 Jul, 2012.

28. Johansson, R., Storm, A., Stephansen, C., Eikedal, S., Willassen, T., Skaug, S., ... \& Perks, D. (2011, February). "A 1/13-inch 30fps VGA SoC CMOS image sensor with shared reset and transfer-gate pixel control". In Solid-State Circuits Conference Digest of Technical Papers (ISSCC), 2011 IEEE International (pp. 414-415).

29. Seo, M. W., Suh, S. H., Iida, T., Takasawa, T., Isobe, K., Watanabe, T., ... \& Kawahito, S. (2012). "A Low-Noise High Intrascene Dynamic Range CMOS Image Sensor With a 13 to 19b Variable-Resolution Column-Parallel FoldingIntegration/Cyclic ADC". Solid-State Circuits, IEEE Journal of, 47(1), 272-283.

30. Koklu, G., Ghaye, J., Beuchat, R., De Micheli, G., Leblebici, Y., \& Carrara, S. (2012, May). "Quantitative comparison of commercial CCD and custom-designed CMOS camera for biological applications". In Circuits and Systems (ISCAS), 2012 IEEE International Symposium on (pp. 2063-2066).

31. Bigas, M., Cabruja, E., Forest, J.,\&Salvi, J.(2006). "Review of CMOS image sensors". Microelectronics journal, 37(5), 433-451.

32. Schöberl, M., Senel, C., Fössel, S., Bloss, H., \& Kaup, A. (2009). "Non-linear Dark Current Fixed Pattern Noise Compensation for Variable Frame Rate Moving Picture Cameras". In Proc. 17th European Signal Processing Conference (EUSIPCO) (pp. 268-272).

33. Otsu, N. (1975). "A threshold selection method from gray-level histograms". Automatica, 11(285-296), 23-27.

34. Song, B., Sivagnanam, V., Vandevyver, C. D., Hemmila, I., Lehr, H. A., Gijs, M. A., \& Bünzli, J. C. G. (2009). "Time-resolved lanthanide luminescence for lab-on-a-chip detection of biomarkers on cancerous tissues". Analyst, 134(10), 1991-1993. 
35. Ciftlik, A. T., Song, B., Vandevyver, C., Bünzli, J. C., Lehr, H. A., \& Gijs, M. (2010). "Fast Immunohistochemical BioMarker Detection Device for Cancer Tissue Slices". In Proc. of 14 th Int. Conf. on Miniaturized Systems for Chem. and Life Sc. (MicroTAS) (pp. 699-70).

36. Hilgers, Allen R., Robert A. Conradi, and Philip S. Burton. "Caco-2 cell monolayers as a model for drug transport across the intestinal mucosa." Pharmaceutical research 7.9 (1990): 902-910.

37. Vergeres, Guy, et al. "The NutriChip project translating technology into nutritional knowledge." British Journal of Nutrition 1.1 (2012): 1-7.

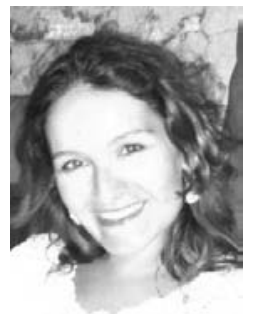

Gözen Köklü received the B.S. degree in Microelectronics Engineering Program of Sabanci University (SU), Istanbul, Turkey in 2007. In March 2009, she received the MSc degree from the Electronical Engineering Department ofEcolePolytechnique Federale de Lausanne (EPFL), Lausanne, Switzerland. She is currently working toward a Ph.D. degree at the Integrated Systems Laboratory (LSI) and Microelectronics Sytems Laboratory (LSM) at EPFL. Her main area of interest during her master degree was design and fabrication of biosensor chips, silicon nanowire transistors and Analog to Digital Converters. Her current research topic is developing a highly dedicated CMOS image detection system for Nutrition Analysis as part of one of NutrciChip project granted by the Swiss Nano-Tera.ch initiative.

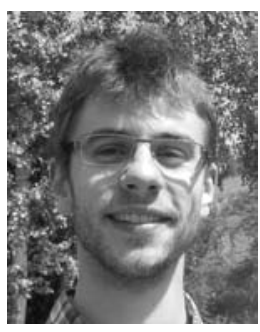

Julien Ghaye received a M.Sc. in electrical engineering (microelectronics) from EPFL, Lausanne, Switzerland, in 2009 and a M.Sc. in electrical engineering (Information Technologies) from the Université de Liège, Belgium in 2010. Since 2011, he is pursuing a Ph.D. in electrical engineering at the Integrated Systems Laboratory (LSI) at EPFL. During the course of his master's projects, he got acquainted with RTL design and verification, image processing and embedded systems. His research interest focuses on the development and hardware integration of image and signal processing for bio-sensing applications.

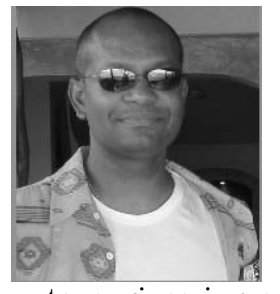

RalphEtienne-Cummings(S'94-M'98SM'08) received theB.Sc. degreein physics from Lincoln University, Oxford, PA, in 1988, and the M.S.E.E. and Ph.D. degrees in electrical engineering from the University of Pennsylvania, Philadelphia, in 1991 and 1994, respectively. Currently, he is a Professor of electrical and computer engineering, and computer science at The Johns Hopkins University (JHU), Baltimore, MD. He is the former Director of Computer Engineering at JHU and the Institute of Neuromorphic Engineering, currently administered by the University of Maryland, College Park. He was also an Associate Director for Education and Outreach of the National Science Foundation (NSF) sponsored Engineering Research Centers on Computer Integrated Surgical Systems and Technology at JHU. Dr. Etienne-Cummings is the recipient of the NSF CAREER Award and the Office of Naval Research Young Investigator Program Award. In 2006, he was named a Visiting African Fellow and a Fulbright Fellowship Grantee for his sabbatical at the University of Cape Town, Rondebosch, South Africa.
He is currently an Eminent Visiting Scholar to the U. Western Sydney, Sydney Australia and was elected IEEE Fellow in 2012. His current research interests include mixed-signal very large scale integration systems, computational sensors, computer vision, neuromorphic engineering, smart structures, mobile robotics, legged locomotion, and neuroprosthetic devices.

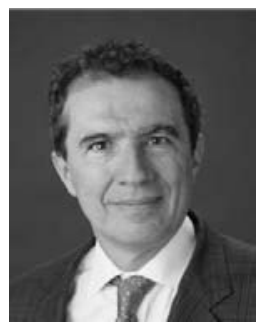

Giovanni De Micheli (F'94) is Professor and Director of the Institute of Electrical Engineering and of the Integrated Systems Centre, EPFL, Lausanne, Switzerland. He is Program Leader of the Nano-Tera.ch program. His research interests include several aspects of design technologies for integrated circuits and systems, such as synthesis for emerging technologies, networks on chips, and 3-D integration. He is also interested in heterogeneous platform design, including electrical components and biosensors as well as in data processing of biomedical information. Prof. De Micheli is the recipient of the 2003 IEEE Emanuel Piore Award for contributions to computer-aided synthesis of digital systems. He is a Fellow of ACM. He received the Golden Jubilee Medal for outstanding contributions to the IEEE CAS Society in 2000. He received the 1987 D. Pederson Award for the best paper on the IEEE TRANSACTIONS ON COMPUTERAIDED DESIGN/ICAS; two Best Paper Awards at the Design Automation Conference in 1983 and in 1993; and a Best Paper Award at the DATE Conference in 2005. He has served IEEE in several capacities, namely, Division 1 Director (20082009), Co-Founder and President Elect of the IEEE Council on EDA (2005-2007), President of the IEEE CAS Society (2003), and Editor-in-Chief of the IEEE TRANSACTIONS ON COMPUTER-AIDED DESIGN/ICAS (1987-2001). He is and has been chair of several conferences, including DATE (2010), pHealth (2006), VLSI SOC (2006), DAC (2000), and ICCD (1989).

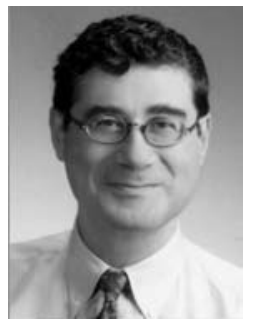

Yusuf Leblebici (F'10) received the B.Sc. and M.Sc. degrees in electrical engineering from Istanbul Technical University, Istanbul, Turkey, in 1984 and 1986, respectively, and the Ph.D. degree in electrical and computer engineering from the University of Illinois, Urbana-Champaign (UIUC), in 1990. Between 1991 and 2001, he worked as a faculty member at UIUC, at Istanbul Technical University, and at Worcester Polytechnic Institute (WPI). In 2000-2001, he also served as the Microelectronics Program Coordinator at Sabanci University. Since 2002, he has been a Chair Professor at the Swiss Federal Institute of Technology in Lausanne (EPFL), and director of Microelectronic Systems Laboratory. His research interests include design of high-speed CMOS digital and mixed-signal integrated circuits, computer-aided design of VLSI systems, intelligent sensor interfaces, modeling and simulation of semiconductor devices, and VLSI reliability analysis. He is the coauthor of four textbooks, namely, HotCarrier Reliability of MOS VLSI Circuits (Kluwer Academic Publishers, 1993), CMOS Digital Integrated Circuits: Analysis and Design (McGraw Hill, 1st Edition 1996, 2nd Edition 1998, 3rd Edition 2002), CMOS Multichannel Single-Chip Receivers for Multi-Gigabit Optical Data Communications (Springer, 2007) and Fundamentals of High Frequency CMOS Analog Integrated Circuits (Cambridge University Press, 2009), as well as more than 200 articles published in various journals and conferences. Dr. Leblebici has served as an Associate Editor 
of IEEE TRANSACTIONS ON CIRCUITS AND SYSTEMS (II) and IEEE TRANSACTIONS ON VERY LARGE SCALE INTEGRATION (VLSI) SYSTEMS. He has also served as the general co-Chair of the 2006 European Solid-State Circuits Conference, and the 2006 European Solid State Device Research Conference (ESSCIRC/ESSDERC). He has been elected as Distinguished Lecturer of the IEEE Circuits and Systems Society for 2010-2011.

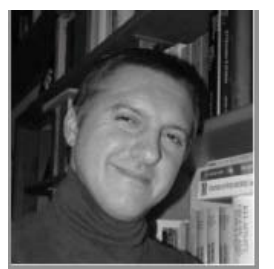

Sandro Carrara(M'08) is a lecturer and scientist at the EPFL in Lausanne (Switzerland) and professor of optical and electrical biosensors at the Department of Electrical Engineering and Biophysics (DIBE) of the University of Genoa (Italy). $\mathrm{He}$ is founder and Editor-in-Chief of the journalBioNanoScience by Springer, Topical Editor of the IEEE Sensors Journal, and Associate Editor of IEEE Transactions on Biomedical Circuits and Systems. He is an IEEE member for the Circuit and System Society (CASS) and has been recently appointed as CASS representative to the IEEE Sensors Council. He also has been recently appointed as CASS Distinguished Lecturers for the years 2013-2014. His scientific interests are on electrical phenomena of nano-biostructured films, and include CMOS design of biochips based on proteins and DNA. He has more then 120 scientific publications and 10 patents. His work received a NATO Advanced Research Award in 1996 for the original contribution to the physics of single-electron conductivity in nano-particles, two Best Paper Awards at the IEEE PRIME Conference in 2010 (Berlin), and in 2009 (Cork), a Best Poster Award at the Nanotera workshop in 2011 (Bern), and a Best Poster Award at the NanoEurope Symposium in 2009 (Rapperswil). He also received the Best Referees Award from the journal Biosensor and Bioelectronics in 2006 . 